Published in final edited form as:

Nature. 2017 February 09; 542(7640): 255-259. doi:10.1038/nature21061.

\title{
Molecular mechanism for the regulation of yeast separase by securin
}

\author{
Shukun Luo ${ }^{1}$ and Liang Tong ${ }^{1}$ \\ ${ }^{1}$ Department of Biological Sciences, Columbia University, New York, NY 10027, USA
}

\section{Abstract}

Separase has a critical role in dissolving the cohesion among sister chromatids during chromosome segregation ${ }^{1-7}$. Separase is over-expressed in human tumors, making it a potential target for drug discovery ${ }^{8}$. The protease activity of separase is strictly regulated by the inhibitor securin, which forms a tight complex with separase and may also stabilize this enzyme ${ }^{9-16}$. Separases are large, 140-250 kD enzymes, with an N-terminal a-helical region and a caspase-like catalytic domain $(\mathrm{CD})$ at the $\mathrm{C}$-terminus. While crystal structures of the $\mathrm{C}$-terminal two domains of separase ${ }^{17}$ and low-resolution electron microscopy reconstructions of the separase-securin complex ${ }^{18,19}$ have been reported, the atomic structures of full-length separase and especially the complex with securin are not known. Here we report crystal structures at up to $2.6 \AA$ resolution of the yeast Saccharomyces cerevisiae separase-securin complex. The a-helical region of separase (also known as Esp1) contains four domains (I-IV), and a substrate-binding domain (SD) immediately precedes the $\mathrm{CD}$ and has tight associations with it. The separase-securin complex assumes a highly elongated structure. Residues $258-373$ of securin (Pds1), named the separase interaction segment (SIS), is primarily in an extended conformation and traverses the entire length of separase, having interactions with all of its domains. Most importantly, residues 258-269 of securin are located in the separase active site, illuminating its mechanism of inhibition. Biochemical studies confirm the structural observations and indicate that contacts outside the separase active site are crucial for stabilizing the complex, thereby defining an important function for the helical region of separase.

Saccharomyces cerevisiae separase contains 1630 amino acid residues and the N-terminal ahelical region covers $\sim 1160$ residues. We obtained good quality crystals for the complex of S. cerevisiae separase (residues 51-1630; Fig. 1a, Extended Data Figs. 1-3) and securin (residues 258-373; Extended Data Fig. 4). The N-terminal segment of securin contains the KEN- and D-box motifs, which are important for the destruction of securin but not for

\footnotetext{
Users may view, print, copy, and download text and data-mine the content in such documents, for the purposes of academic research, subject always to the full Conditions of use: http://www.nature.com/authors/editorial_policies/license.html\#termsReprints and permissions information is available at www.nature.com/reprints.

Correspondence information for Liang Tong, Phone: (212) 854-5203, FAX: (212) 865-8246, 1tong@ columbia.edu.

Author Contributions. SL carried out cloning, protein expression, purification, crystallization, data collection, structure determination and refinement, and site-directed mutagenesis. LT initiated the project, supervised the entire research, and analyzed the results. SL and LT wrote the paper.

The authors declare no competing financial interests.

Readers are welcome to comment on the online version of the paper.
} 
interaction with separase ${ }^{20}$. The structure was determined and refined at 3.0 A resolution (Extended Data Table 1). Under the same crystallization condition, we observed another crystal form and collected a data set to $3.7 \AA$ resolution. We readily solved the structure of this crystal form and did not observe any significant differences.

We produced crystals of another complex of $S$. cerevisiae separase (residues 71-1630) and securin (residues 258-373), and determined its structure at $2.6 \AA$ resolution. The overall structure of this complex is essentially the same as that at 3.0 A resolution (Extended Data Fig. 5). One difference is that residues 75-79 at the N-terminal end of separase form an antiparallel $\beta$-sheet with the equivalent residues of another separase molecule in the crystal (Extended Data Fig. 5), which is an artifact due to the longer truncation of separase in this complex.

The overall structure of the separase-securin complex has a highly elongated shape, with dimensions of $65 \AA \times 70 \AA \times 165 \AA$ (Figs. 1b, 1c). The CD is located at one end of the complex, far away from the N-terminus of separase at the other end. This elongated shape is generally consistent with that observed for the human separase-securin complex by $\mathrm{EM}^{18}$, but distinct from a closed form reported for the C. elegans complex ${ }^{19}$.

The structure shows that the N-terminal helical region (residues 53-1163) of separase can be divided into four domains (I-IV) (Figs. 1a-c). Their structures are generally like HEAT repeats, with extensions that connect each domain to the next one. Domain I is folded back onto domain II such that the loops at one end of each domain are facing each other (Fig. 2a), with a buried surface area of $1,100 \AA^{2}$ for each domain, suggesting that this may be a stable association between them. Two consecutive helices (the fourth and fifth helices) in domain I are parallel to each other (Fig. 2a, Extended Data Fig. 6). The first 50 residues of separase are poorly conserved (Extended Data Fig. 1). Earlier studies showed that the first 155 residues of separase are important for function ${ }^{12}$. These residues include helices a 1 to a 5 of domain I. Their deletion would disrupt the structure of this domain and its interaction with domain II (Fig. 2a), and helix a 3 is in the binding site for the C-terminal helix of securin (see below), thereby explaining their functional importance. Human separase is stimulated by DNA of greater than $100 \mathrm{bp}^{21}$, and this stimulation is likely also mediated by the helical region.

The helical hairpins of domain III are arranged in a tight, right-handed super-helix (Fig. 2b). This domain is located in the middle of the structure and has contacts with domain II at one end (buried surface area of $\left.\sim 900 \AA^{2}\right)$ and domain IV at the other $\left(\sim 1,100 \AA^{2}\right)$ (Fig. 1b). It also has a direct contact with a segment immediately following the catalytic Cys 1531 residue in the $\mathrm{CD}$ (strands $\beta 4 \mathrm{~A}, \beta 4 \mathrm{~B}$ and the connecting loop, Fig. 2c, Extended Data Figs. 3,6 ). The overall arrangement of the helices in this domain has similarity to that in several other structures based on a DaliLite search ${ }^{22}$, and the closest homologs include the TPR domain of the G-protein signaling modulator LGN ${ }^{23-25}$ and others (Extended Data Fig. 7).

Domain IV has extensive interactions with the domain immediately prior to the CD (SD, Fig. 1a; see next), covering one face of its $\beta$-sheet (buried surface area of $\sim 1,900 \AA^{2}$ for each domain) and providing one extra strand to this $\beta$-sheet (Fig. 2c). It also interacts with two of 
the surface helices of the CD. Therefore, domain IV could be considered as forming a module together with the SD-CD.

An $a+\beta$ domain is located just prior to the $C D$ in separases ${ }^{18,26}$. The backbone fold of this domain has no similarity to caspases ${ }^{17}$ or other proteases. The domain is tightly associated with the $\mathrm{CD}$ and contributes to substrate binding. We will refer to it as the substrate-binding domain (SD) here (Fig. 1a). Other names for the SD and CD include PPD (pseudo-protease domain) and APD (active protease domain), respectively ${ }^{17}$. The SD contains a five-stranded $(\beta 1-\beta 5)$ mostly anti-parallel $\beta$-sheet, and there is a large insertion (residues 1200-1380) between the third and fourth strands, including a hairpin of two long helices $(a \mathrm{~A}-\alpha \mathrm{B})$ and a four-helical bundle ( $\mathrm{aC}-\mathrm{aF}$ ) (Fig. 2c). The backbone fold of this $\beta$-sheet is RNase H-like, with close homologs including a part of the catalytic PIWI domain of Argonaute ${ }^{27}$ and others (Extended Data Fig. 7).

The overall structure of the SD-CD of $S$. cerevisiae separase is similar to that of the $C$. thermophilum separase ${ }^{17}$ (Fig. 2c), with rms distance of $1.5 \AA$ for their equivalent $\mathrm{Ca}$ atoms and amino acid sequence identity of 39\%, suggesting that binding of securin did not cause a large overall conformational change in these two domains. The $\beta$-sheets of the two domains are juxtaposed in the structure, although the two neighboring strands at the interface ( $\beta 5$ of SD and $\beta 6$ of $C D$ ) are at an angle of nearly $90^{\circ}$. The aA-aB hairpin from SD covers a portion of the active site in $\mathrm{CD}$ and helps mediate substrate binding.

Securin is primarily in an extended conformation, with only two short helices, and runs along the entire length of separase (Figs. 1b, 3a). We will refer to this segment of securin, residues 258-373, as the separase interaction segment (SIS). The backbone direction of SIS is anti-parallel to that of separase, such that the N-terminal region of SIS interacts with the C-terminal region of separase. The securin SIS has contacts with every domain of separase, being positioned in prominent grooves in its surface (Fig. 3a). Approximately 4,600 $\AA^{2}$ of the surface area of securin is buried in the interface with separase $\left(\sim 3,900 \AA^{2}\right.$ surface area burial), indicating the tight association and the stability of this complex.

Most importantly, residues 258-269 of the securin SIS, with good electron density (Fig. 3b), are located in the active site of separase, interacting with the SD and CD (Figs. 3a, 3c). Therefore, one mechanism for securin to inhibit the protease activity of separase is through blocking substrate access to the active site. Residues Ile259-Glu260-Ile261 of securin mimic the $\mathrm{P}_{5}-\mathrm{P}_{3}$ residues of the substrate ${ }^{17}$ (Fig. 3c). Especially, the side chain of Ile259 has hydrophobic interactions with Trp1249 and Trp1250 located near the tip of the aA-aB helical hairpin of SD, and that of Glu260 is positioned near the N-terminus of helix aA in CD. However, the $\mathrm{P}_{1}$ Arg residue of the substrate is replaced with Pro263 in securin. It assumes a different conformation and its main-chain carbonyl is $6 \AA$ away from the thiol group of the catalytic Cys1531 residue (Figs. 3c, 3d). The position of Pro263 also clashes with that of His 2083 in the $C$. thermophilum $\mathrm{CD}^{17}$, the second member of the catalytic machinery, and the conformation of the equivalent His1505 in the securin complex is probably an inactive one (Fig. 3d). These differences ensure that securin is not cleaved by separase even though it is located in the active site. Earlier studies showed that mutations of 
residues of securin equivalent to the $\mathrm{P}_{2}, \mathrm{P}_{1}$ and $\mathrm{P}_{1}{ }^{\prime}$ residues could turn it into a substrate (Extended Data Fig. 8) ${ }^{14,17 .}$

Residues 264-269 of securin have interactions with CD, and the $\mathrm{P}^{\prime}$ side of the substrate may be recognized by this region of separase as well. In contrast to the sequence conservation of the $\mathrm{P}$ side of the substrate, the $\mathrm{P}^{\prime}$ side is much less conserved (Extended Data Fig. 8). A $\mathrm{P}_{1}{ }^{\prime}$ Arg residue, found in several fungal species but not in $S$. pombe or $C$. thermophilum, may mediate the degradation of this fragment by the $\mathrm{N}$-end rule pathway ${ }^{28}$. The structure shows that a groove in the active site region can accommodate the $\mathrm{P}^{\prime}$ residues, and the $\beta 4 \mathrm{~A}-\beta 4 \mathrm{~B}$ hairpin following the catalytic Cys1531 may contribute to substrate binding here (Extended Data Fig. 8; see below). Residues 264-269 of securin are in contact with the rim of this groove rather than being located at its bottom, possibly due to the shift in the position of the Pro263 residue relative to that of the $\mathrm{P}_{1}$ Arg of the substrate (Fig. 3c).

Following the active site region, residues 271-288 of the securin SIS are in close contact with domain III of separase (Fig. 3e), and residues 290-296 are located in a deep groove at the interface between domains III and II (Figs. 3a, Extended Data Fig. 6). These interactions are mostly hydrophobic in nature, and also involve several aromatic (Phe and Tyr) residues. Residues 306-316 of the securin SIS are placed on the side of domain II of separase (Fig. 1c). Finally, residues 365-373 at the C-terminus of securin form a short helix (Extended Data Fig. 4) and are located in a surface depression in domain I (Fig. 1b, Extended Data Fig. 6). Residues 299-305 and 317-362 of securin are disordered in the current structure.

The securin SIS contains three potential phosphorylation sites (Fig. 1c) ${ }^{29,30}$, Ser277 (Fig. 3e), Ser292 (Extended Data Fig. 6) and Thr304. The S277A/S292A/T304A triple mutant had weaker interaction with separase and reduced nuclear location of separase, while the single and double mutants had no growth phenotype ${ }^{30}$. pSer277 should have favorable interactions with Arg1130 in domain IV of separase (Fig. 3e). The side chain of Ser292 is hydrogen-bonded to the main-chain amides of residues 294 and 295 in the SIS and Thr304 is in a disordered region in the current structure, suggesting possible conformational rearrangements of Ser292 and Thr304 upon phosphorylation.

Separase residues in contact with securin are generally well conserved (Extended Data Figs. 1-3). The securin SIS is poorly conserved among species, but the residues in the major contact sites with separase are better conserved (Extended Data Fig. 4), suggesting that the securin homologs may share a common mechanism of interacting with and inhibiting separase. The observed binding mode of the SIS indicates that securin likely helps to stabilize the overall structure of separase, as suggested by prior biochemical studies ${ }^{12-15}$. It might also be possible that once securin is removed by proteasome degradation the structure of separase alone could undergo a conformational change to assume a different arrangement of its domains, especially for domains I-III. The $\beta 4 \mathrm{~A}-\beta 4 \mathrm{~B}$ segment is a loop (L4) in the structure of the $C$. thermophilum SD-CD (Extended Data Fig. 6), and residues in this loop are important for catalytic activity ${ }^{17}$. This segment is stabilized by contacts with domain III (Extended Data Fig. 6), and therefore a change in the position of domain III might be necessary for catalysis. This would represent another, indirect mechanism for securin to inhibit the catalytic activity of separase. 
The structure of the separase-securin complex provides a molecular basis for the large amount of experimental data on this complex. This structure is also supported by our biochemical observations. The complex from the fungus $Z$ ygosaccharomyces rouxii was used for these studies, and its separase and the SIS of securin are closely related to those from S. cerevisiae (Extended Data Figs. 1-4). Z. rouxii securin contains only 282 residues due to a shorter $\mathrm{N}$-terminal segment (Fig. $4 \mathrm{a}$ ). We found that separase was produced in the insoluble fraction in insect cells unless an appropriate securin was co-expressed with it (Fig. 4b; Extended Data Fig. 9), which was likely due to the instability/misfolding of separase. Based on this observation, we assessed the importance of various segments of securin for producing soluble separase, which would reflect both its binding and chaperone functions. The N-terminal segment of securin, up to residue 166 (equivalent to residue 258 of $S$. cerevisiae securin, Extended Data Fig. 4), could not help produce soluble separase, and deleting these $165 \mathrm{~N}$-terminal residues had little effect on the production of soluble separase (Figs. 4b, 4c). Additional experiments showed that deleting residue 231 to the C-terminus of securin led to a relatively small reduction in the solubility of separase (Figs. $4 \mathrm{~b}, 4 \mathrm{c}$ ). These data indicate that residues 166-230 of $Z$. rouxii securin, equivalent to residues 258-316 of $S$. cerevisiae securin, are required for interactions with separase, in agreement with our structural information.

We also observed that the segment covering residues 189 to the C-terminus of $Z$. rouxii securin was able to produce a small amount of soluble separase (Figs. 4b, 4c). This segment would be missing the residues located in the active site of separase, suggesting that binding to the active site is not essential for complex formation with separase. On the other hand, gel-filtration studies of this complex showed that it migrated in the void volume, indicating that it was mostly aggregated. Therefore, even though this segment of securin could bind separase, it might not be sufficient for its chaperone function to promote the correct separase folding and/or conformation.

The segment covering residues $166-188$ of $Z$. rouxii securin was not able to help produce soluble separase, indicating that binding to the active site region of separase alone is not sufficient to produce a stable complex and that the $\mathrm{N}$-terminal helical region of separase has a crucial role in the regulation by securin. In addition, residues 189-250 of securin produced a much lower amount of soluble separase compared to that for residues 189-282 of securin, indicating an appreciable role for the $\mathrm{C}$-terminal residues of securin.

Overall, our studies have produced the first crystal structure information on the separasesecurin complex and defined the molecular basis for the tight regulation of separase by securin. The structure provides a foundation for understanding the biochemical and biological functions of this complex from $S$. cerevisiae. While the N-terminal helical region is poorly conserved among separases at the amino acid sequence level, they are better conserved at the secondary structure level (Extended Data Figs. 1-2). Therefore, it might be possible that the separase-securin complexes from other organisms could assume a similar overall architecture as observed here, which is supported by the similar shape for the human separase-securin complex ${ }^{18}$. The information obtained from this structure may also be useful for deciphering the molecular mechanisms of other separase-securin complexes. 


\section{Online Methods}

\section{Protein expression and purification}

Separase and securin were co-expressed in insect cell system using Multibac technology (Geneva Biotech) ${ }^{31}$. Separase and securin gene fragments were cloned into the pFL dual plasmid through its $\mathrm{PH}$ and p10 promoters, respectively. The construct used for crystallization contained residues 51-1630 or 71-1630 of Saccharomyces cerevisiae separase and residues $258-373$ of securin. A hexa-histidine tag was added to the $\mathrm{N}$ terminus of separase while securin did not carry a tag. Bacmids were generated by transforming constructs to DH10EMBacY competent cells (Geneva Biotech) and isolated by Bac-to-Bac protocol (ThermoFisher). Bacmids were further transfected into Sf9 cells using Cellfectin II (ThermoFisher) following the manufacturer's instructions to produce P1 baculoviruses. P2 viruses were obtained by amplifying P1 viruses in Sf9 once and were used for large-scale protein expression.

High5 cells were grown in Fernbach flasks (PYREX) in ESF-921 medium (Expression System) by shaking at $120 \mathrm{rpm}$ at $27^{\circ} \mathrm{C}$ until the density reaches $2 \times 10^{6}$ cells $/ \mathrm{ml}$. Cells were infected by $\mathrm{P} 2$ virus and harvested 48 hours after infection by centrifugation at $500 \times \mathrm{g}$. The harvested cells were resuspended in lysis buffer containing $50 \mathrm{mM}$ phosphate ( $\mathrm{pH} 7.6)$, $500 \mathrm{mM} \mathrm{NaCl}, 5 \%$ (v/v) glycerol, $20 \mathrm{mM}$ imidazole and protease inhibitor cocktail (Sigma) and lysed by sonication. Cell lysates were centrifuged at $24,000 \times \mathrm{g}$ for $40 \mathrm{~min}$ at $4{ }^{\circ} \mathrm{C}$ before incubating with nickel beads (Qiagen). After $1 \mathrm{~h}$, beads were transferred to a gravity flow column (Biorad) and washed extensively with lysis buffer. Protein was eluted with a buffer containing $50 \mathrm{mM}$ phosphate (pH 7.6), $500 \mathrm{mM} \mathrm{NaCl}, 5 \%$ (v/v) glycerol and $500 \mathrm{mM}$ imidazole. Protein eluate was further purified by gel filtration using Sephacryl S-300 column (GE Healthcare) equilibrated in a buffer containing $5 \mathrm{mM}$ HEPES (pH 7.6), $400 \mathrm{mM} \mathrm{NaCl}$ and $2 \mathrm{mM}$ DTT. The protein samples were concentrated to $10 \mathrm{mg} / \mathrm{ml}$ and stored at $-80^{\circ} \mathrm{C}$.

\section{Protein crystallization}

Crystals were grown using the sitting-drop method at $20{ }^{\circ} \mathrm{C}$ by mixing $1.0 \mu \mathrm{l}$ protein solution at $3 \mathrm{mg} / \mathrm{ml}$ concentration with $1.0 \mu \mathrm{l}$ well solution containing $0.25 \mathrm{M} \mathrm{Na} / \mathrm{K}$ phosphate (pH 6.0) and 14\% (w/v) polyethylene glycol 3,350. Crystals appeared in 3 days and were transferred to the well solution plus $30 \%(\mathrm{v} / \mathrm{v})$ glycerol as cryoprotectant before being flash-frozen in liquid nitrogen for data collection at $100 \mathrm{~K}$. For phasing, a gold derivative was obtained by soaking crystals in mother liquor with $2 \mathrm{mM} \mathrm{KAu}(\mathrm{CN})_{2}$ for 5 hours and transferring to cryoprotectant for $2 \mathrm{~min}$. The crystals belong to space group $P 3_{2} 21$ with one separase-securin complex in the asymmetric unit.

\section{Data collection and structure determination}

X-ray diffraction data sets up to $3.0 \AA$ resolution were collected at beamline 24-ID-E of the Advanced Photon Source (APS). The data were processed with the HKL package ${ }^{32}$. The structure was solved using the single isomorphous replacement with anomalous scattering method with the gold derivative with AutoSharp ${ }^{33}$, which located 10 gold sites. The atomic model was built manually with Coot ${ }^{34}$ and refined with PHENIX ${ }^{35}$. The crystallographic information is summarized in Extended Data Table 1. 
From the same crystallization condition, crystals belonging to space group $C 2$ were also obtained, with unit cell parameters of $a=238.4 \AA, b=89.5 \AA, c=119.0 \AA$ and $\beta=105.2^{\circ}$. There is one separase-securin complex in the asymmetric unit. The best diffraction data set that we could collect on this crystal form extended to 3.7 A resolution. The structure could be readily solved with the molecular replacement method using the structure in $P 3_{2} 21$ as the model, and no significant structural differences were observed.

X-ray diffraction data sets for the crystal containing residues 71-1630 of separase were collected at beamline 24-ID-C of the Advanced Photon Source (APS). The structure was refined at 2.6 ̊ resolution, and the crystallographic information is summarized in Extended Data Table 1.

For the $2.6 \AA$ structure, $94.8 \%$ of the residues are in the favored region of the Ramachandran plot. For the $3.0 \AA$ structure, $96.9 \%$ of the residues are in the favored region of the Ramachandran plot. No residues are in the disallowed region in either structure.

\section{Mutagenesis studies}

Site-specific and deletion mutations were introduced with the QuikChange kit (Agilent) and sequenced for confirmation. The mutants were expressed in insect cells following the same protocol as that for the wild-type protein.

\section{Data availability}

The atomic coordinates and the diffraction data sets have been deposited at the Protein Data Bank, with accession codes 5U1S and 5U1T. 


\section{Extended Data}

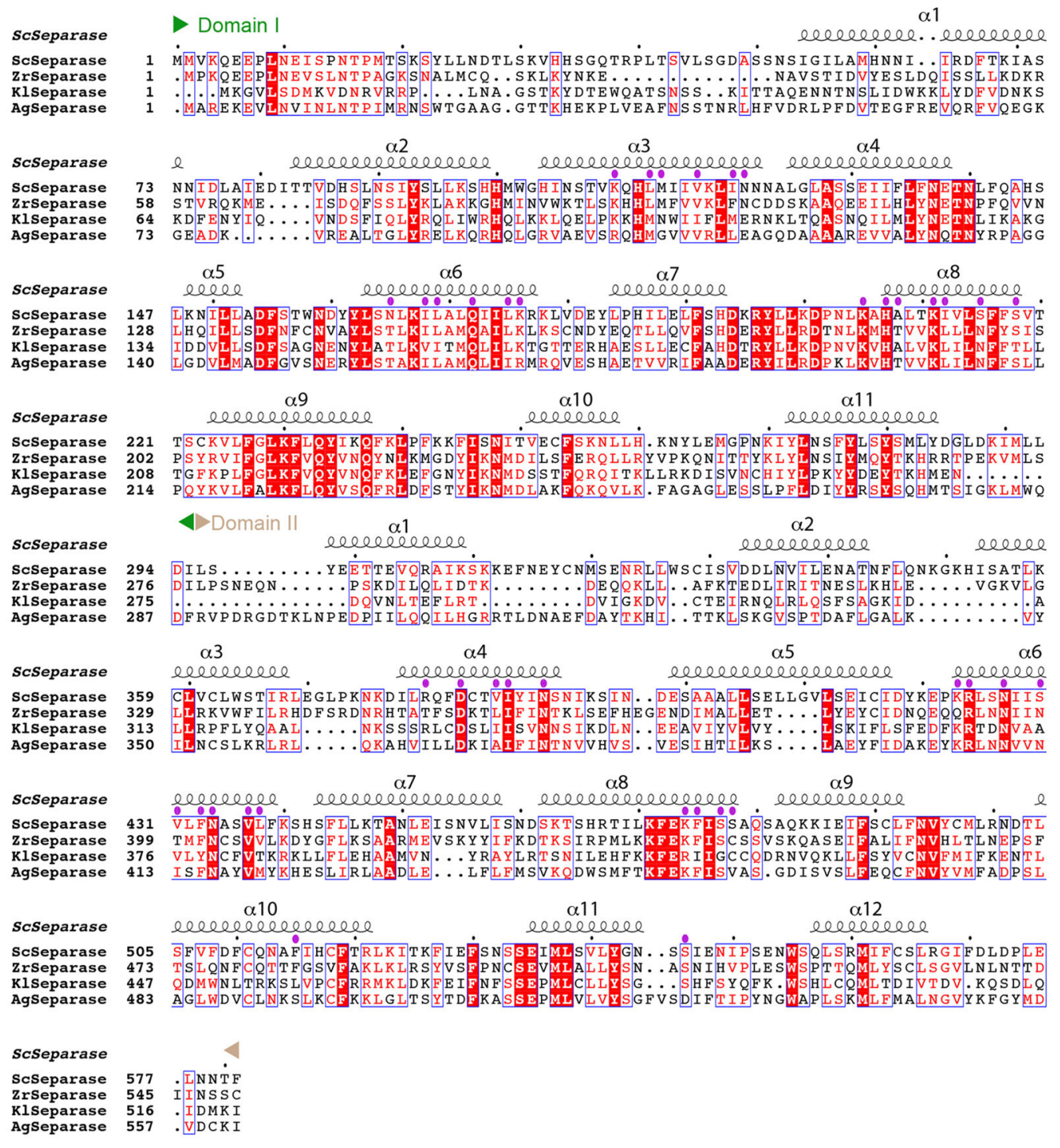

Extended Data Fig. 1.

Sequence alignment of domains I and II of separase. The secondary structure elements in the $S$. cerevisiae separase structure are shown and labeled. The boundaries of the domains are indicated. Residues in contact with securin are indicated with the purple dots. Sc:

Saccharomyces cerevisiae, Zr: Zygosaccharomyces rouxii, $\mathrm{Kl}$ : Kluyveromyces lactis; Ag: Ashbya gossypii. Modified from an output from ESPript ${ }^{36}$. 


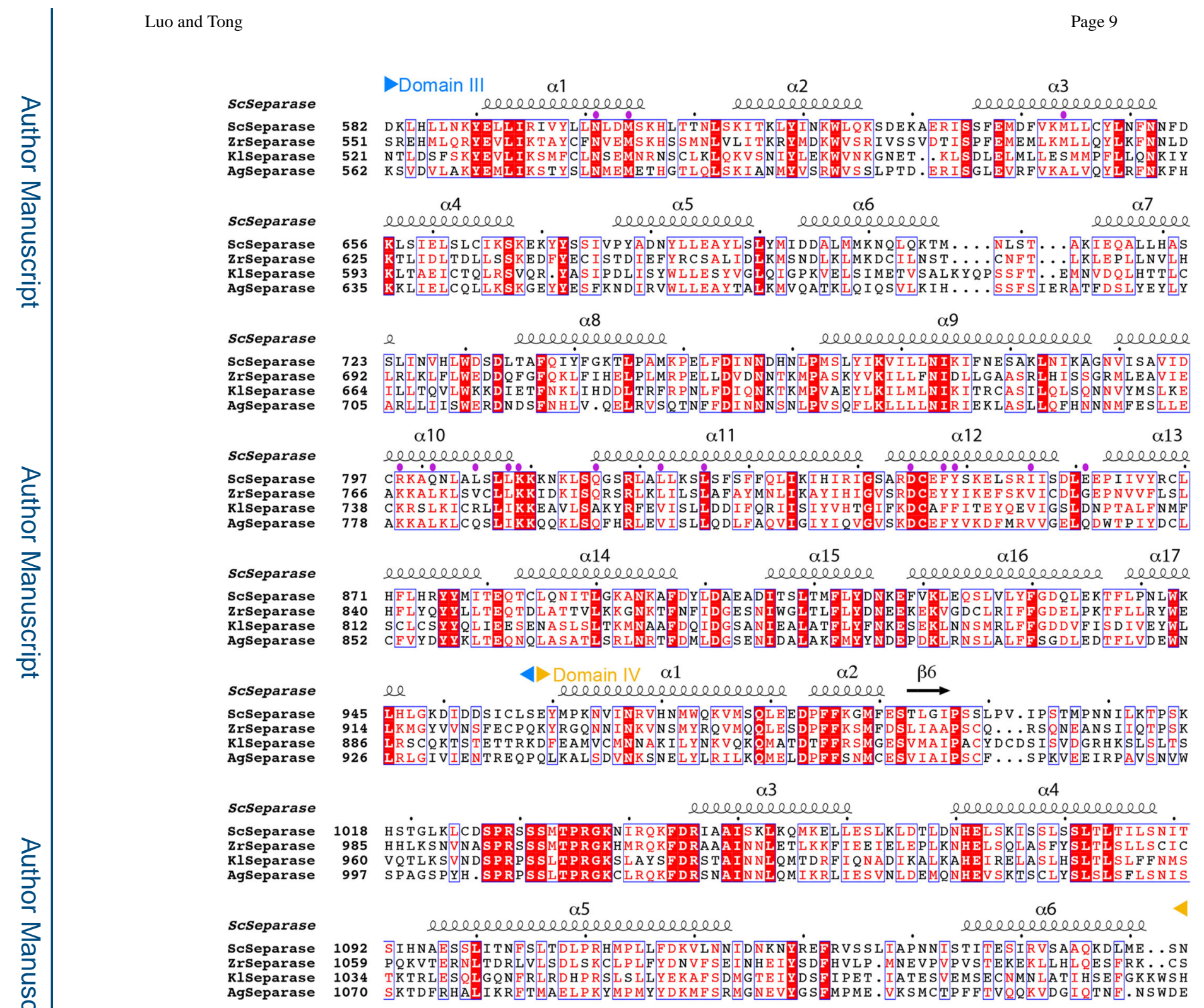

Extended Data Fig. 2.

Sequence alignment of domains III and IV of separase. The secondary structure elements in the $S$. cerevisiae separase structure are shown. 


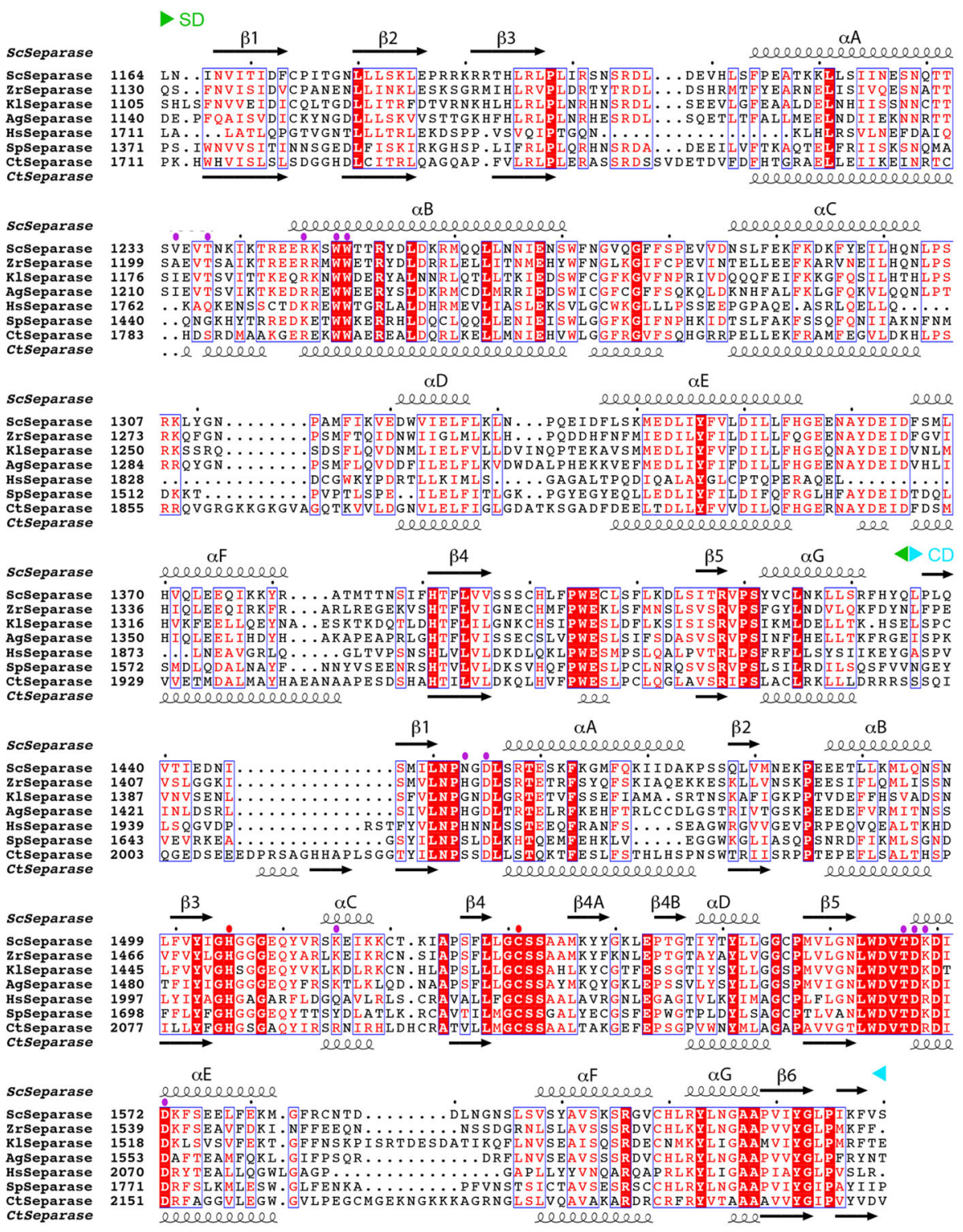

Extended Data Fig. 3.

Sequence alignment of the SD and CD of separase. The catalytic Cys1531 and His1505 residues are indicated with the red dots. Hs: Homo sapiens, Sp: Schizosaccharomyces pombe; Ct: Chaetomium thermophilum. 


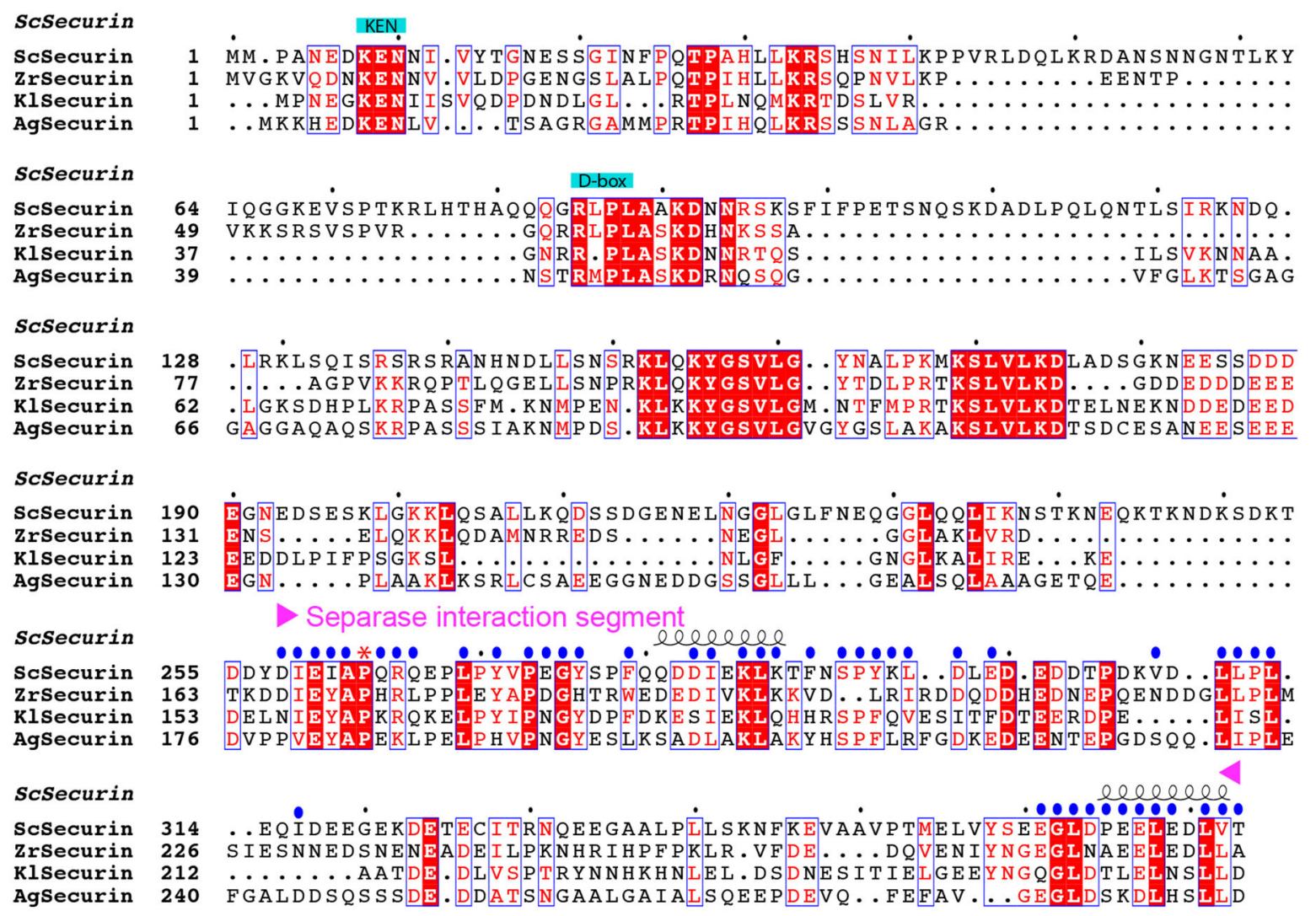

Extended Data Fig. 4.

Sequence alignment of securin. The separase interaction segment (SIS) is indicated.

Residues in contact with separase are indicated with the blue dots. Residue 263 is equivalent to the $\mathrm{P}_{1}$ residue of separase substrates, and is indicated with the red asterisk. Residues 317360 of $S$. cerevisiae securin are disordered in the current structures and are poorly conserved in sequence. 

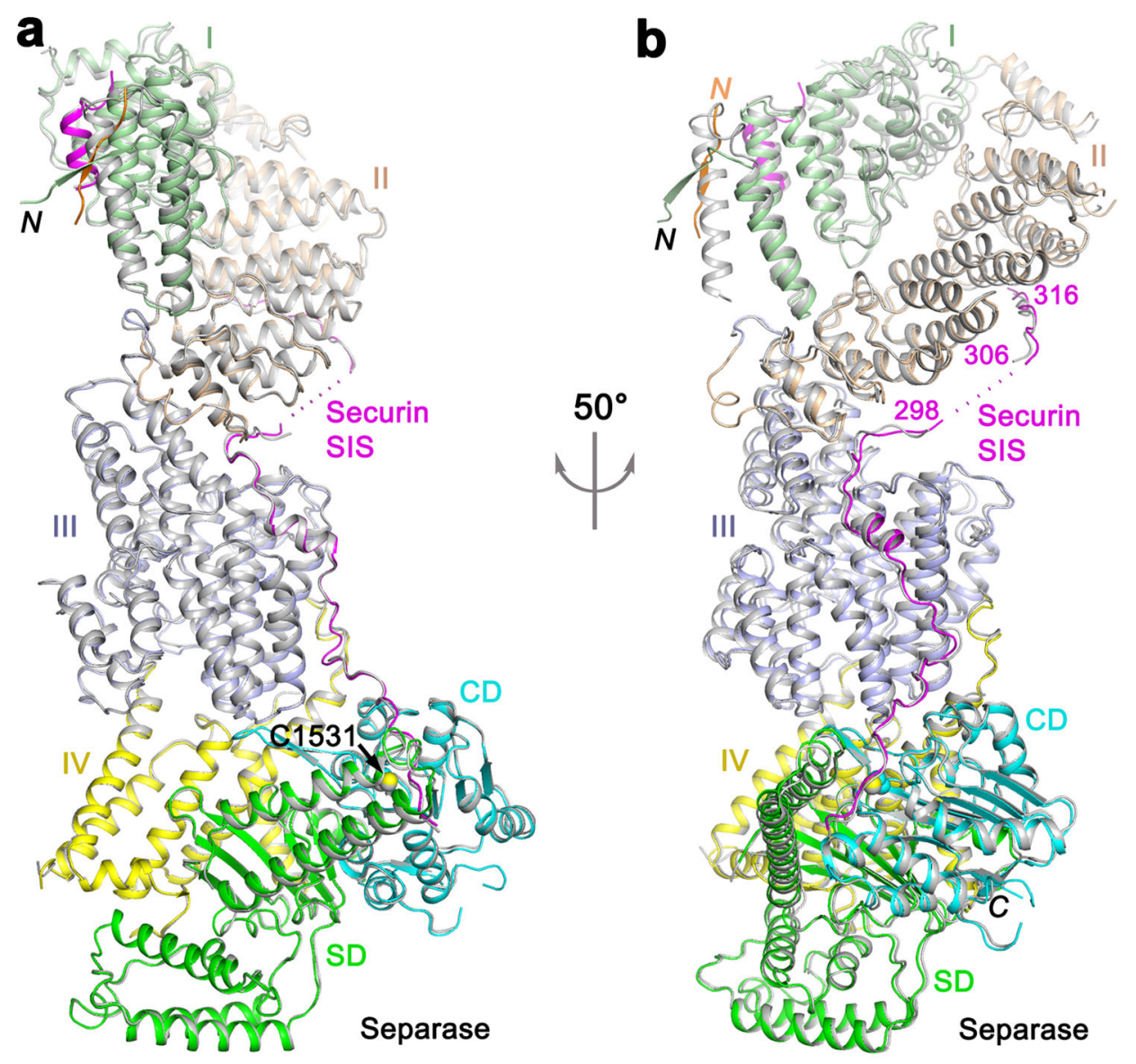

Extended Data Fig. 5.

Overlay of the structures of the separase-securin complexes. (a). The complex formed by residues 71-1630 of separase and 258-373 of securin is shown in color, and that by residues 51-1630 of separase and 258-373 of securin in gray. Residues 73-80 from another molecule of separase in the crystal is shown in orange, forming a $\beta$-sheet with the $\mathrm{N}$-terminal segment of separase. (b). panel a viewed after a $50^{\circ}$ rotation around the vertical axis. 
a
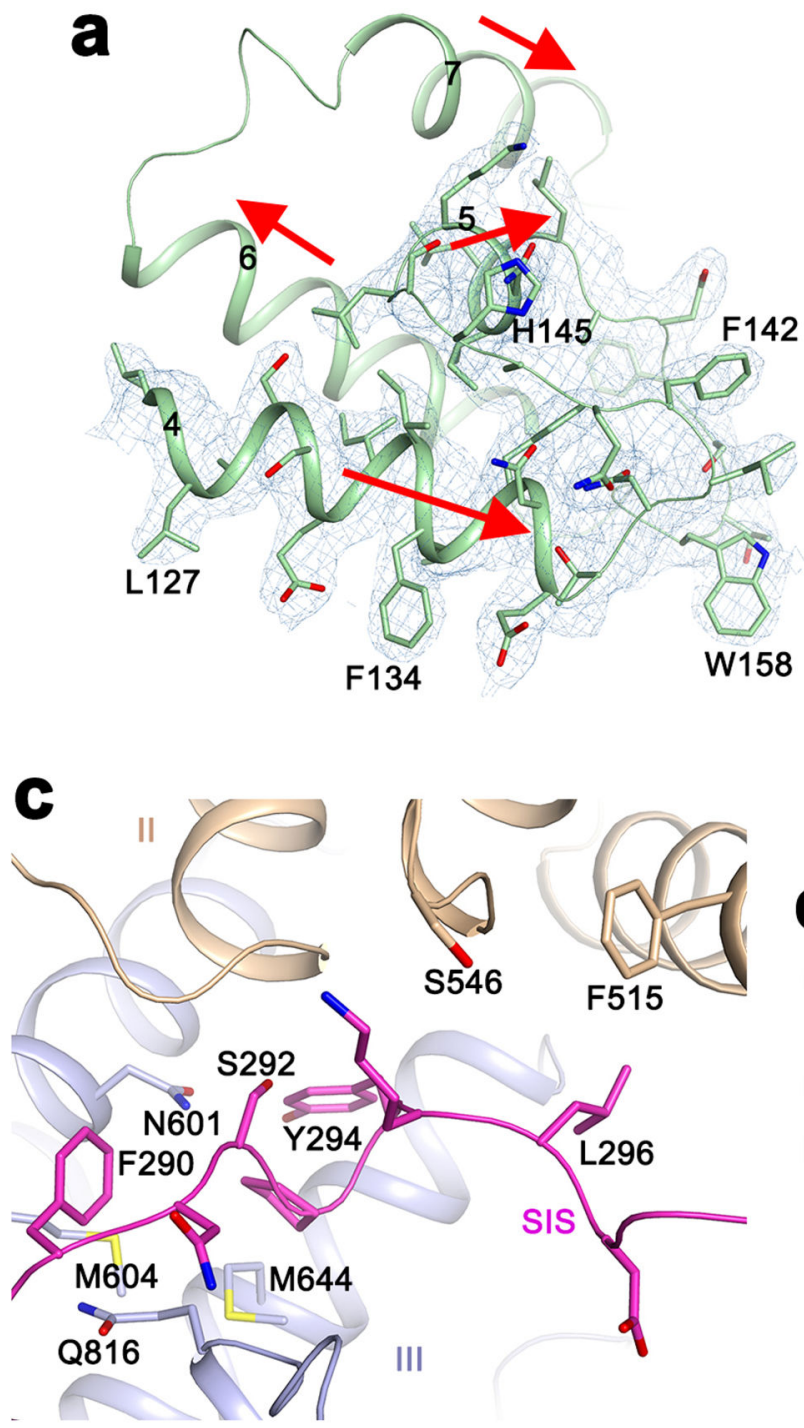

b
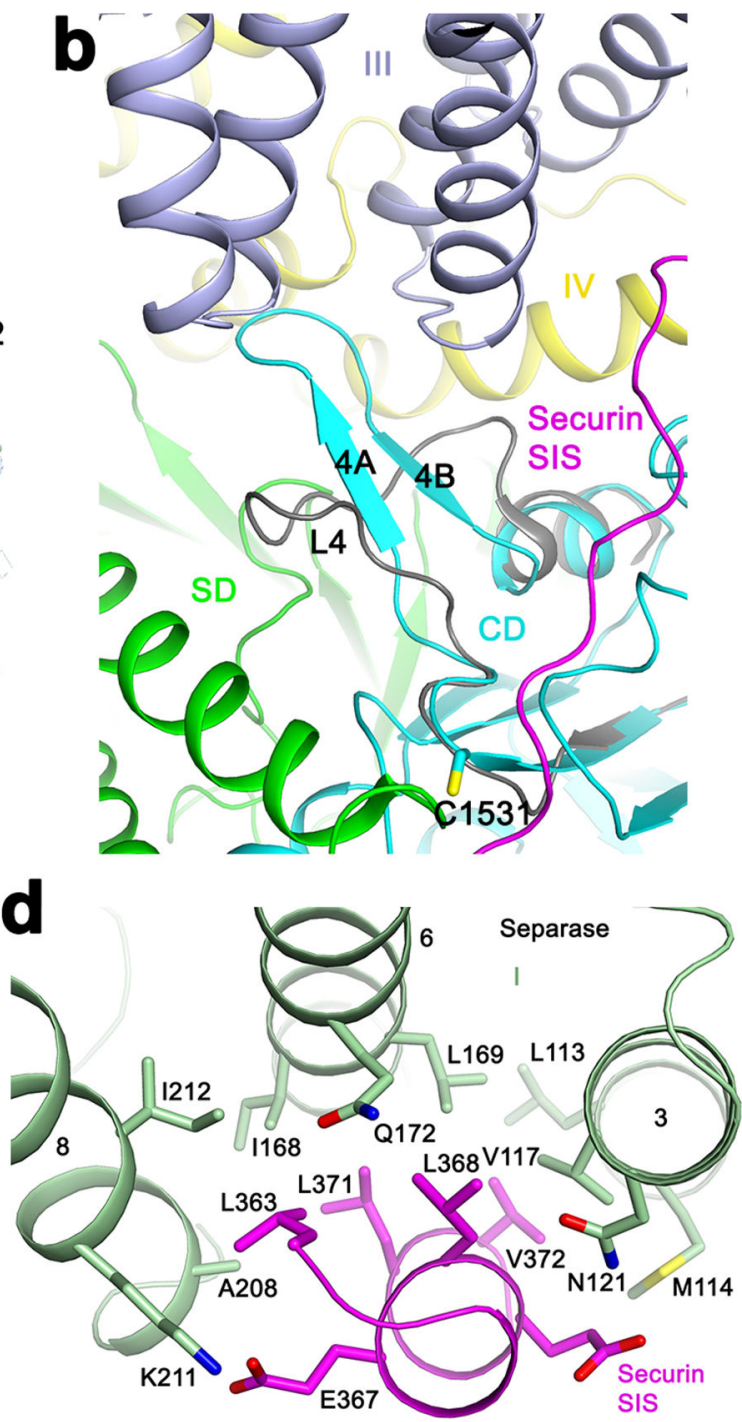

Extended Data Fig. 6.

Additional structural information on the separase-securin complex. (a). $2 \mathrm{~F}_{\mathrm{o}}-\mathrm{F}_{\mathrm{c}}$ electron density for helices 4 and 5 of domain I at $2.6 \AA$ resolution, contoured at $1 \sigma$. Helices 6 and 7 are also shown for reference. The directions of the helices are indicated with the red arrows. (b). The $\beta 4 \mathrm{~A}-\beta 4 \mathrm{~B}$ segment of $\mathrm{CD}$ (cyan) is immediately after the catalytic Cys 1531 residue, and has interactions with domain III (light blue). The equivalent segment in the $C$. thermophilum SD-CD free enzyme structure is a loop (L4), shown in dark gray. (c). Interactions between residues 290-296 of securin SIS (magenta) with domains III (light blue) and II (light brown) of separase. (d). Interactions between the C-terminal segment of securin SIS (magenta) and domain I of separase (green). Deletion of the first 155 residues of separase ${ }^{12}$ would remove helix a 3 in this binding site. 

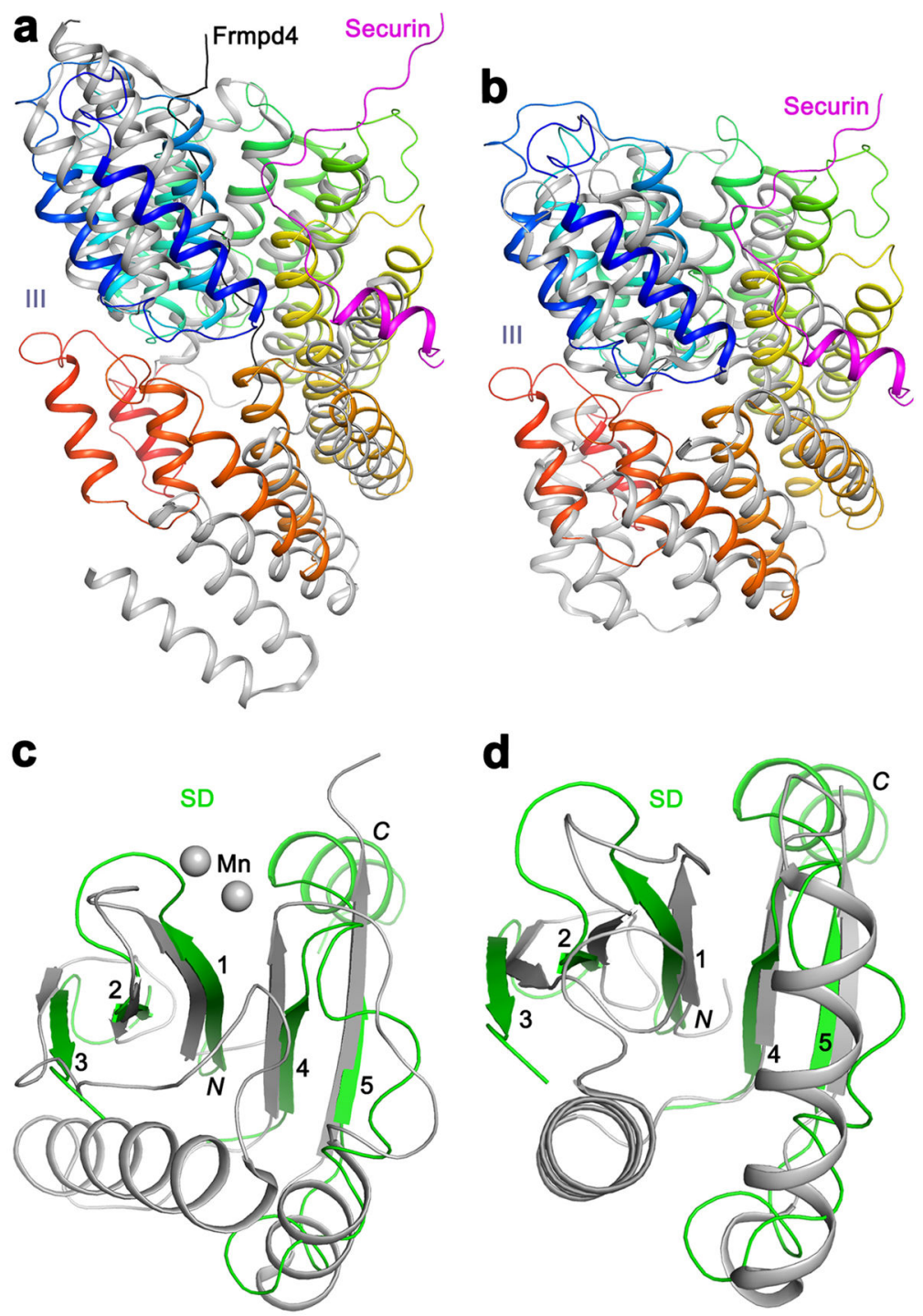

Extended Data Fig. 7.

Structural homologs of domains III and SD of separase. (a). Overlay of the structures of domain III of separase (color ramp from N- (blue) to C-terminus (red)) and the TPR domain of LGN (gray, PDB entry $4 \mathrm{WNG} ; 11 \%$ sequence identity, $Z$ score of 14.4 ) ${ }^{23-25}$. The Frmpd4 ligand (black) of LGN is bound to a different region of the structure compared to securin. (b). Overlay of the structures of domain III of separase and the subunit 7 of the APC/C (PDB entry 5G04; 5\% identity, $14.3 Z$ score) ${ }^{37}$. (c). Overlay of the structures of the SD of separase (green) and a part of the PIWI domain of Argonaute (gray, PDB entry 4N76; $10 \%$ sequence identity, $5.5 Z$ score. As a comparison, matching this $\beta$-sheet to that in $C$. thermophilum separase produced a $Z$ score of 5.7.) ${ }^{27,38}$. Residues in the helical insert between $\beta 3$ and $\beta 4$ of separase are removed for clarity. (d). Overlay of the structures of the SD of separase and the YqgF domain of Tex (gray, PDB entry 3BZK; 4\% sequence identity, $5.5 Z$ score) ${ }^{39}$. 

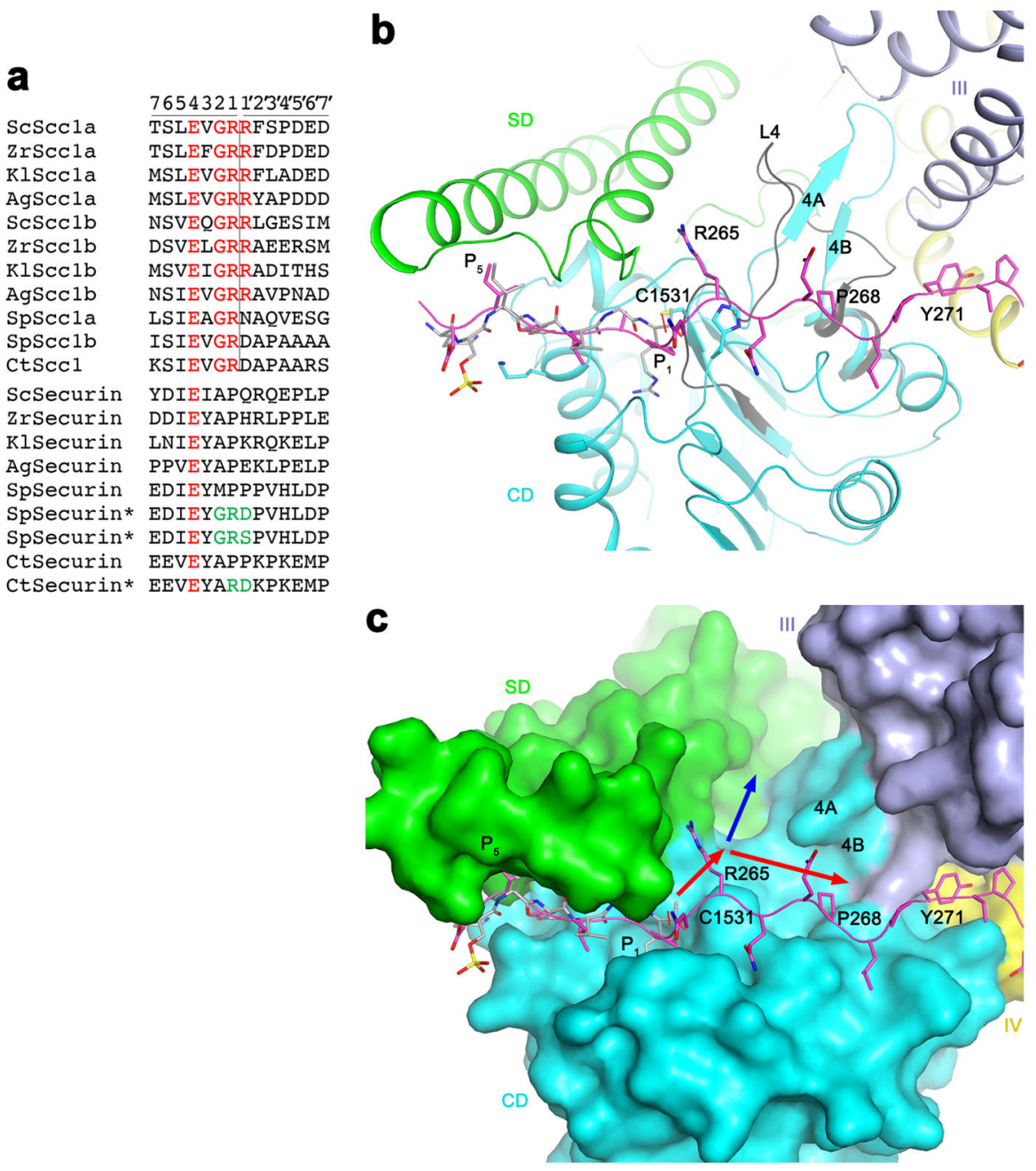

\section{Extended Data Fig. 8.}

Possible binding groove for the $\mathrm{P}^{\prime}$ residues of the substrate. (a). Alignment of the separase cleavage sites in Scc1 substrates. The two cleavage sites in each protein are named a and $b$. The equivalent residues in securin are also shown. The asterisks indicate securin mutants (mutations in green) that become substrates of separase. The $\mathrm{P}$ and $\mathrm{P}^{\prime}$ residues are labeled at the top, and the cleavage site is indicated with the vertical line. (b). The overall binding mode of residues $258-271$ of securin in separase. The $\beta 4 \mathrm{~A}-\beta 4 \mathrm{~B}$ segment of $\mathrm{CD}$ (cyan) is a loop (L4, dark gray) in the $C$. thermophilum SD-CD structure. (c). A groove in the active site of separase (red arrows) can accommodate the $\mathrm{P}^{\prime}$ residues. The blue arrow indicates another groove in this region, but the binding mode of securin suggests that the groove indicated by the red arrows is more likely. 


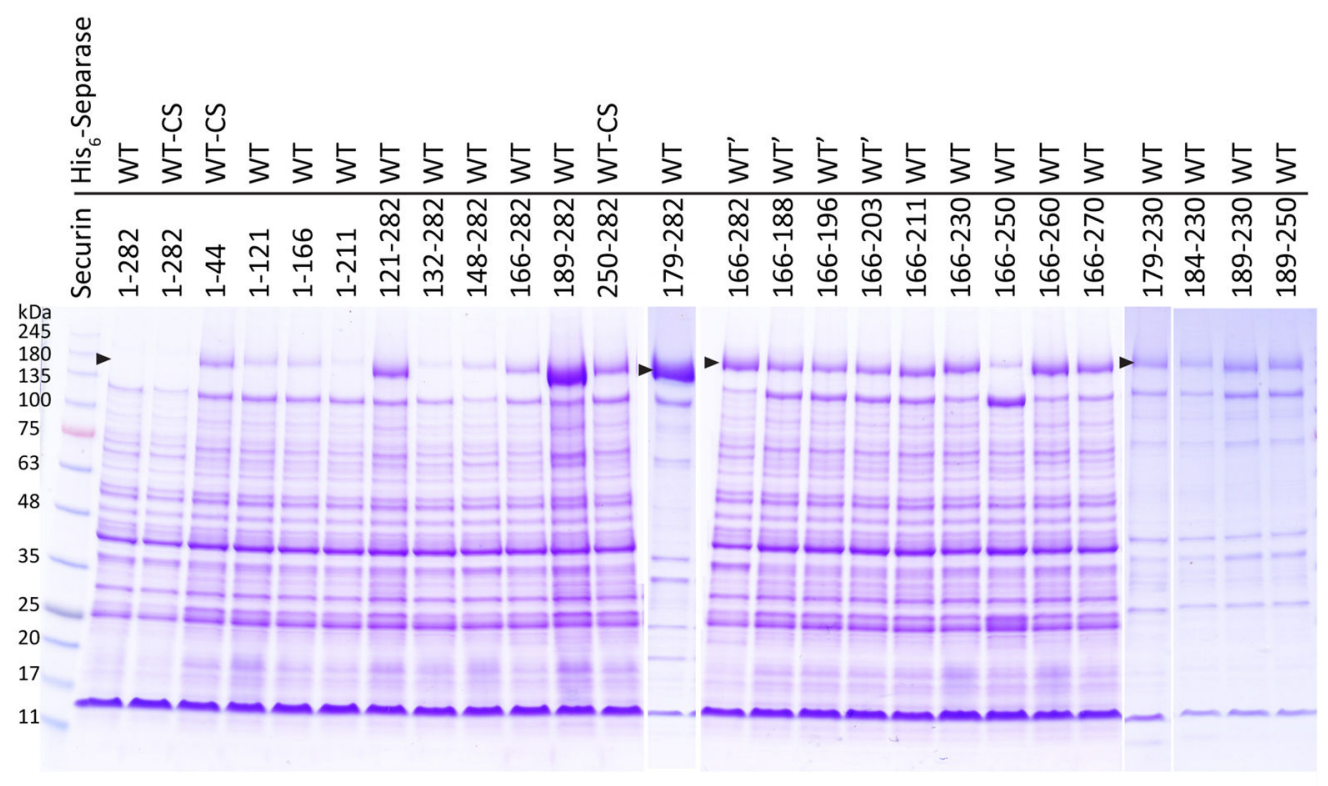

\section{Extended Data Fig. 9.}

Biochemical characterizations of the interactions between separase and securin. $Z$. rouxii separase was co-expressed with various segments of $Z$. rouxii securin, with truncations at the $\mathrm{N}$ - and/or C-terminus. The insoluble fraction was run on SDS PAGE. The position of separase (with an N-terminal His tag) is indicated with the black arrowhead. WT: full-length $Z$. rouxii separase; WT-CS: full-length $Z$. rouxii separase with $\mathrm{C} 1497 \mathrm{~S}$ mutation; $\mathrm{WT}^{\prime}: Z$. rouxii separase with an internal deletion of residues 952-1010, corresponding to a poorly conserved, disordered loop in domain IV of the $S$. cerevisiae separase structure. For gel source data, see Supplementary Figure 1.

\section{Extended Data Table 1}

Data collection and refinement statistics

\begin{tabular}{|c|c|c|c|}
\hline & $\begin{array}{l}\text { Separase (51-1630)-securin } \\
(258-373) \text { complex crystal } \\
\text { form I }\end{array}$ & $\begin{array}{l}\text { Separase (71-1630)-securin } \\
(258-373) \text { complex crystal } \\
\text { form I }\end{array}$ & $\begin{array}{l}\text { Separase }(51-1630)- \\
\text { securin }(258-373) \\
\text { complex crystal form } \\
\text { II }\end{array}$ \\
\hline \multicolumn{4}{|l|}{ Data collection } \\
\hline Space group & $P 3_{2} 21$ & $P 3_{2} 21$ & $C 2$ \\
\hline \multicolumn{4}{|l|}{ Cell dimensions } \\
\hline$a, b, c(\AA)$ & $126.3,126.3,273.9$ & $125.9,125.9,271.9$ & $238.4,89.5,119.0$ \\
\hline$a, \beta, \gamma\left({ }^{\circ}\right)$ & $90,90,120$ & $90,90,120$ & $90,105.2,90$ \\
\hline Resolution ( & $50-3.0(3.11-3.0)^{*}$ & $50-2.6(2.69-2.6)^{*}$ & $50-3.7(3.93-3.7)$ \\
\hline$R_{\text {merge }}(\%)$ & $14.4(>100)$ & $14.5(>100)$ & $16.6(232)$ \\
\hline $\mathrm{CC}_{1 / 2}$ & $(0.480)$ & $(0.476)$ & $(0.271)$ \\
\hline $\mathrm{I} / \sigma \mathrm{I}$ & $15.0(1.5)$ & $14.4(1.2)$ & $4.43(0.49)$ \\
\hline Completeness (\%) & $99.8(99.6)$ & $100(99.9)$ & $96.2(90.9)$ \\
\hline Redundancy & $10.4(7.6)$ & $10.0(8.2)$ & $2.7(2.5)$ \\
\hline
\end{tabular}




\begin{tabular}{|c|c|c|c|}
\hline & $\begin{array}{l}\text { Separase }(51-1630) \text {-securin } \\
(258-373) \text { complex crystal } \\
\text { form I }\end{array}$ & $\begin{array}{l}\text { Separase (71-1630)-securin } \\
(258-373) \text { complex crystal } \\
\text { form I }\end{array}$ & $\begin{array}{l}\text { Separase }(51-1630) \text { - } \\
\text { securin }(258-373) \\
\text { complex crystal form } \\
\text { II }\end{array}$ \\
\hline \multicolumn{4}{|l|}{ Refinement } \\
\hline Resolution $(\AA)$ & $50-3.0(3.11-3.0)$ & $50-2.6(2.69-2.6)$ & \\
\hline No. reflections & $51,130(4,818)$ & $77,428(7,588)$ & \\
\hline$R_{\text {work }} / R_{\text {free }}(\%)$ & $19.5(31.4) / 25.2(36.3)$ & $22.1(34.6) / 26.4(37.0)$ & \\
\hline \multicolumn{4}{|l|}{ No. atoms } \\
\hline Protein & 12,609 & 12,633 & \\
\hline Ligand/ion & 0 & 0 & \\
\hline Water & 0 & 16 & \\
\hline \multicolumn{4}{|l|}{ B-factors } \\
\hline Protein & 97.1 & 72.0 & \\
\hline Ligand/ion & - & - & \\
\hline Water & - & 63.2 & \\
\hline \multicolumn{4}{|l|}{ R.m.s deviations } \\
\hline Bond lengths $(\AA)$ & 0.003 & 0.011 & \\
\hline Bond angles $\left({ }^{\circ}\right)$ & 0.65 & 1.2 & \\
\hline
\end{tabular}

Two crystals were used for data collection for crystal form I, and one crystal for crystal form II.

Highest resolution shell is shown in parenthesis.

\section{Supplementary Material}

Refer to Web version on PubMed Central for supplementary material.

\section{Acknowledgments}

We thank Hailong Zhang, Song Xiang and Luis Cunha for carrying out initial studies on this project; S. Banerjee, K. Perry, R. Rajashankar, J. Schuermann, N. Sukumar for access to NE-CAT 24-C and 24-E beamlines at the Advanced Photon Source. This research is supported by grants R35GM118093 and S10OD012018 from the NIH to LT. This work is based upon research conducted at the Northeastern Collaborative Access Team beamlines, funded by the NIH (P41 GM103403). The Pilatus 6M detector on 24-ID-C beam line is funded by a NIH-ORIP HEI grant (S10 RR029205). This research used resources of the Advanced Photon Source, a U.S. Department of Energy (DOE) Office of Science User Facility operated by Argonne National Laboratory under Contract No. DEAC02-06CH11357.

\section{References}

1. Uhlmann F, Wernic D, Poupart MA, Koonin EV, Nasmyth K. Cleavage of cohesin by the CD clan protease separin triggers anaphase in yeast. Cell. 2000; 103:375-386. [PubMed: 11081625]

2. Hauf S, Waizenegger IC, Peters JM. Cohesin cleavage by separase required for anaphase and cytokinesis in human cells. Science. 2001; 293:1320-1323. [PubMed: 11509732]

3. Nasmyth K. Segregating sister genomes: the molecular biology of chromosome separation. Science. 2002; 297:559-565. [PubMed: 12142526]

4. Peters JM. The anaphase-promoting complex: proteolysis in mitosis and beyond. Mol Cell. 2002; 9:931-943. [PubMed: 12049731]

5. Uhlmann F. Separase regulation during mitosis. Biochem Soc Trans. 2003; 70:243-251.

6. Yanagida M. Cell cycle mechanisms of sister chromatid separation; role of Cut $1 /$ separin and Cut $2 /$ securin. Genes to Cells. 2000; 5:1-8. [PubMed: 10651900] 
7. Sullivan M, Hornig NCD, Porstmann T, Uhlmann F. Studies on substrate recognition by the budding yeast separase. J Biol Chem. 2004; 279:1191-1196. [PubMed: 14585836]

8. Zhang N, et al. Identification and characterization of separase inhibitors (Sepins) for cancer therapy. J Biomol Screen. 2014; 19:878-889. [PubMed: 24525869]

9. Ciosk R, et al. An ESP1/PDS1 complex regulates loss of sister chromatid cohesion at the metaphase to anaphase transition in yeast. Cell. 1998; 93:1067-1076. [PubMed: 9635435]

10. Zou H, McGarry TJ, Bernal T, Kirschner MW. Identification of a vertebrate sister-chromatid separation inhibitor involved in transformation and tumorigenesis. Science. 1999; 285:418-422. [PubMed: 10411507]

11. Waizenegger IC, Gimenez-Abian JF, Wernic D, Peters JM. Regulation of human separase by securin binding and autocleavage. Curr Biol. 2002; 12:1368-1378. [PubMed: 12194817]

12. Hornig NCD, Knowles PP, McDonald NQ, Uhlmann F. The dual mechanism of separase regulation by securin. Curr Biol. 2002; 12:973-982. [PubMed: 12123570]

13. Jallepalli PV, et al. Securin is required for chromosomal stability in human cells. Cell. 2001; 105:445-457. [PubMed: 11371342]

14. Nagao K, Yanagida M. Securin can have a separase cleavage site by substitution mutations in the domain required for stabilization and inhibition of separase. Genes to Cells. 2006; 11:247-260. [PubMed: 16483313]

15. Wirth KG, et al. Separase: a universal trigger for sister chromatid disjunction but not chromosome cycle progression. J Cell Biol. 2006; 172:847-860. [PubMed: 16533945]

16. Csizmok V, Felli IC, Tompa P, Banci L, Bertini I. Structural and dynamic characterization of intrinsically disordered human securin by NMR spectroscopy. J Amer Chem Soc. 2008; 130:16873-16879. [PubMed: 19053469]

17. Lin Z, Luo X, Yu H. Structural basis of cohesin cleavage by separase. Nature. 2016; 532:131-134. [PubMed: 27027290]

18. Viadiu H, Stemmann O, Kirschner MW, Walz T. Domain structure of separase and its binding to securin as determined by EM. Nat Struct Mol Biol. 2005; 12:552-553. [PubMed: 15880121]

19. Bachmann G, et al. A closed conformation of the Caenorhabditis elegans separase-securin complex. Open Biol. 2016; 6:160032. [PubMed: 27249343]

20. Pfleger CM, Kirschner MW. The KEN box: an APC recognition signal distinct from the D box targeted by Cdh1. Genes Dev. 2000; 14:655-665. [PubMed: 10733526]

21. Sun Y, et al. Separase is recruited to mitotic chromosomes to dissolve sister chromatid cohesion in a DNA-dependent manner. Cell. 2009; 137:123-132. [PubMed: 19345191]

22. Holm L, Kaariainen S, Rosenstrom P, Schenkel A. Searching protein structure databases with DaliLite v.3. Bioinformatics. 2008; 24:2780-2781. [PubMed: 18818215]

23. Takayanagi H, Yuzawa S, Sugimoto H. Structural basis for the recognition of the scaffold protein Frmpd4/Preso1 by the TPR domain of the adaptor protein LGN. Acta Cryst. 2015; F71:175-183.

24. Carminati M, et al. Concomitant binding of Afadin to LGN and F-actin directs planar spindle orientation. Nat Struct Mol Biol. 2016; 23:155-163. [PubMed: 26751642]

25. Pan Z, et al. Structural and biochemical characterization of the interaction between LGN and Frmpd1. J Mol Biol. 2013; 425:1039-1049. [PubMed: 23318951]

26. Winter A, Schmid R, Bayliss R. Structural insights into separase architecture and substrate recognition through computational modelling of caspase-like and death domains. PLoS Comp Biol. 2015; 11:e1004548.

27. Song JJ, Smith SK, Hannon GJ, Joshua-Tor L. Crystal structure of Argonaute and its implications for RISC slicer activity. Science. 2004; 305:1434-1437. [PubMed: 15284453]

28. Rao H, Uhlmann F, Nasmyth K, Varshavsky A. Degradation of a cohesin subunit by the N-end rule pathway is essential for chromosome stability. Nature. 2001; 410:955-959. [PubMed: 11309624]

29. Holt LJ, et al. Global analysis of Cdk1 substrate phosphorylation sites provides insights into evolution. Science. 2009; 325:1682-1686. [PubMed: 19779198]

30. Agarwal R, Cohen-Fix O. Phosphorylation of the mitotic regulator Pds1/securin by Cdc28 is required for efficient nuclear localization of Esp1/separase. Genes Dev. 2002; 16:1371-1382. [PubMed: 12050115] 
31. Fitzgerald DJ, et al. Protein complex expression by using multigene baculovirus vectors. Nat Methods. 2006; 3:1021-1032. [PubMed: 17117155]

32. Otwinowski Z, Minor W. Processing of X-ray diffraction data collected in oscillation mode. Method Enzymol. 1997; 276:307-326.

33. Vonrhein C, Blanc E, Roversi P, Bricogne G. Automated structure solution with autoSHARP. Methods Mol Biol. 2007; 364:215-230. [PubMed: 17172768]

34. Emsley P, Cowtan KD. Coot: model-building tools for molecular graphics. Acta Cryst. 2004; D60:2126-2132.

35. Adams PD, et al. PHENIX: building a new software for automated crystallographic structure determination. Acta Cryst. 2002; D58:1948-1954.

36. Gouet P, Courcelle E, Stuart DI, Metoz F. ESPript: analysis of multiple sequence alignments in PostScript. Bioinformatics. 1999; 15:305-308. [PubMed: 10320398]

37. Zhang S, et al. Molecular mechanism of APC/C activation by mitotic phosphorylation. Nature. 2016; 533:260-264. [PubMed: 27120157]

38. Wang Y, et al. Nucleation, propagation and cleavage of target RNAs in Ago silencing complexes. Nature. 2009; 461:754-761. [PubMed: 19812667]

39. Johnson SJ, et al. Crystal structure and RNA binding of the Tex protein from Pseudomonas aeruginosa. J Mol Biol. 2008; 377:1460-1473. [PubMed: 18321528] 


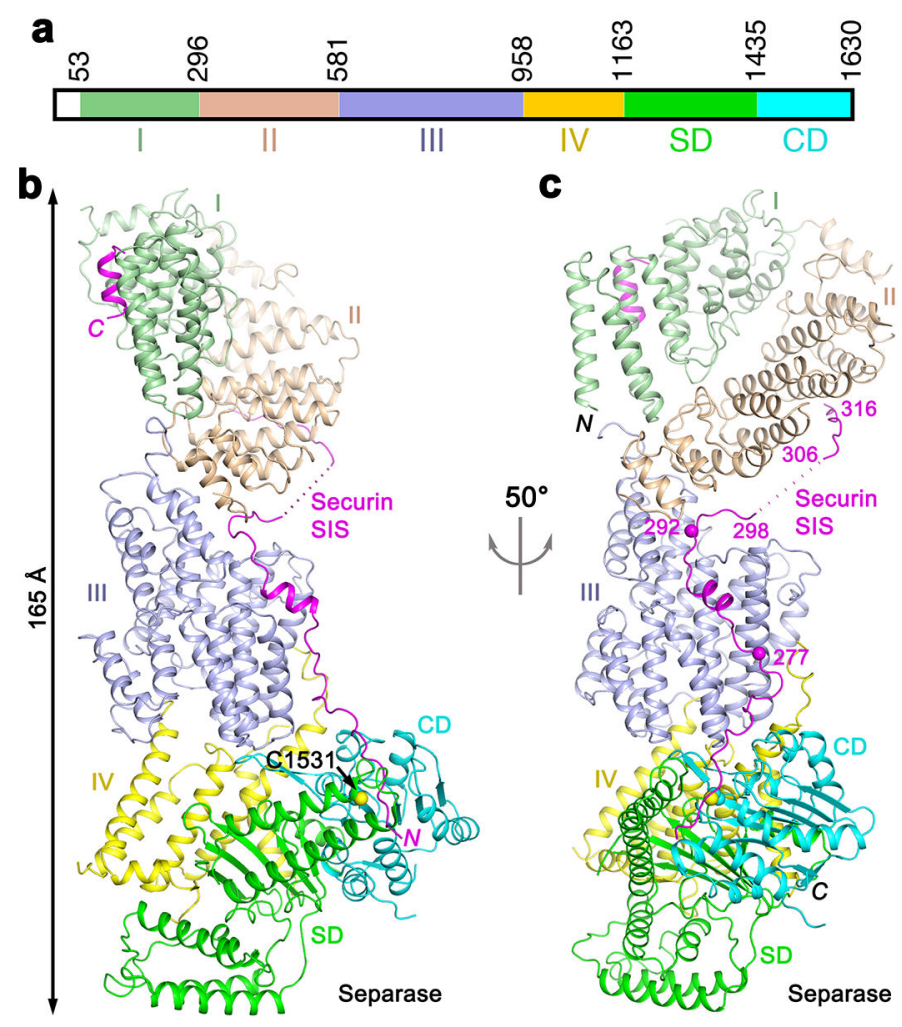

Figure 1.

Crystal structure of the yeast separase-securin complex. (a). Domain organization of $S$. cerevisiae separase. The domains are labeled and given different colors. (b). Overall structure of the yeast separase-securin complex. The domains in separase are colored according to panel a, and the securin SIS is in magenta. The side chain of the catalytic Cys1531 residue of separase is shown as a sphere model. (c). Overall structure of the complex viewed after a $50^{\circ}$ rotation around the vertical axis. Two of the phosphorylation sites in the securin SIS (Ser277 and Ser292) 29,30 are indicated with the spheres and labeled. The structure figures were produced with PyMOL (www.pymol.org). 

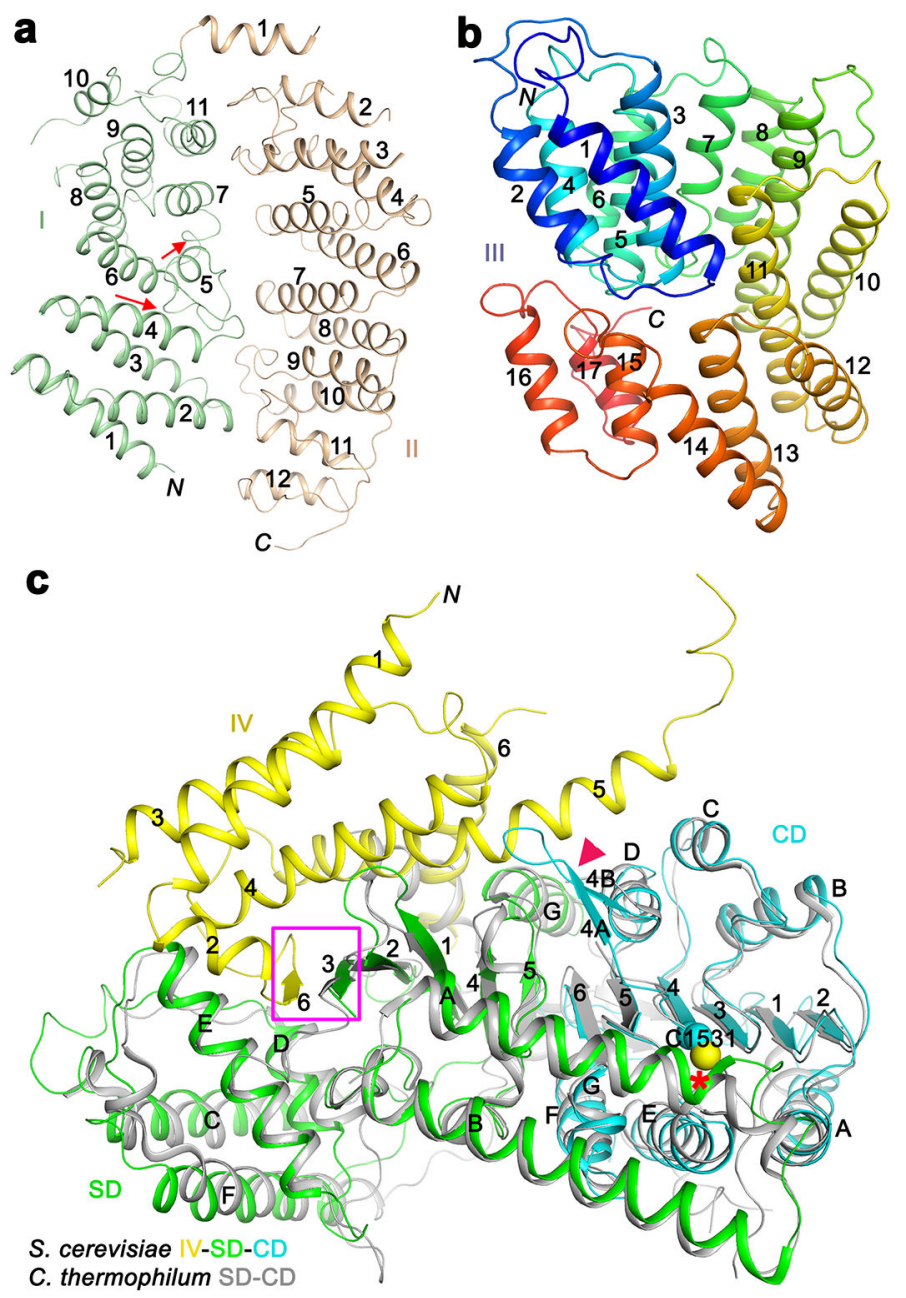

Figure 2.

Structures of the domains in separase. (a). Structure of domains I and II of separase. The directions of helices 4 and 5 in domain I are indicated with the red arrows. (b). Structure of domain III of separase. The helices are colored ramping from blue at the $\mathrm{N}$-terminus to red at C-terminus. (c). Structure of domains IV, SD and CD of separase, and overlay of the structure of the SD-CD of $C$. thermophilum separase (gray) ${ }^{17}$. The active site is indicated with the red asterisk. The red arrowhead indicates the region where the $\beta 4 \mathrm{~A}-\beta 4 \mathrm{~B}$ hairpin has a different conformation and is partly disordered in $C$. thermophilum separase, and the purple box highlights the region where domain IV provides an extra strand (labeled 6) to the $\beta$-sheet of SD. 
a

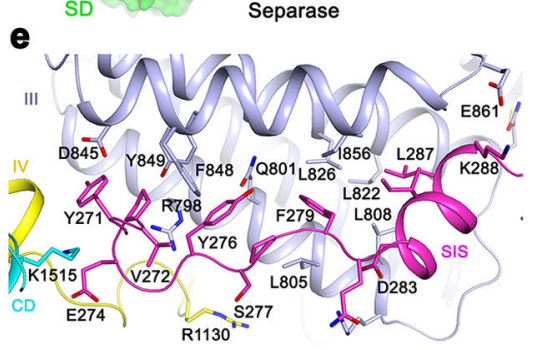

b

C
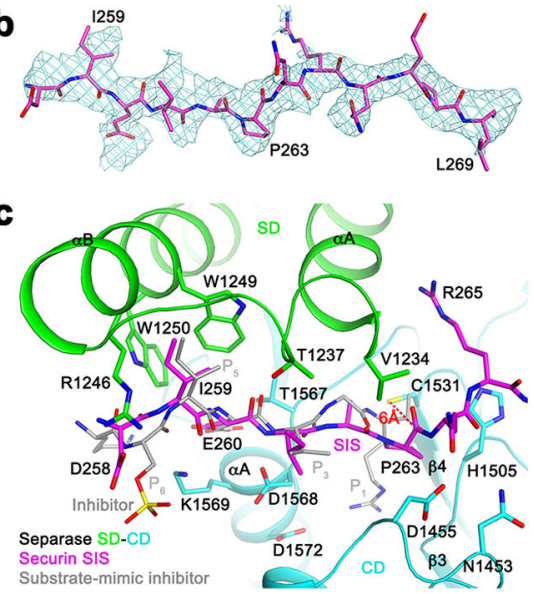

d

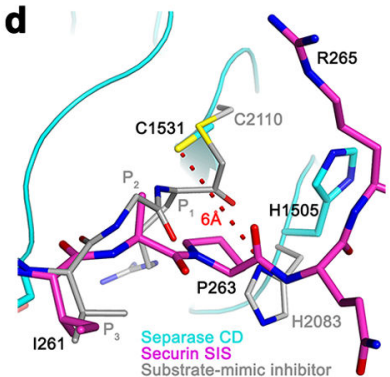

Figure 3.

Interactions between separase and securin. (a). Molecular surface of separase, colored by the domains. The active site of separase is indicated with the red asterisk. The view is same as Fig. 1c. (b). Omit $\mathrm{F}_{\mathrm{o}}-\mathrm{F}_{\mathrm{c}}$ electron density at $3.0 \AA$ A resolution for residues $258-269$ of securin, contoured at $2 \sigma$. (c). Interactions between residues 258-265 of securin SIS (magenta) with the active site of separase. The side chains of residues in the interface are shown as stick models and labeled. The bound position of a substrate-mimic inhibitor to C. thermophilum separase is shown in gray ${ }^{17}$. (d). Close-up of the active site region showing the differences between the bound position of securin (magenta) and that of the inhibitor to $C$.

thermophilum separase (gray) ${ }^{17}$. (e). Interactions between residues $271-288$ of securin SIS (magenta) with domain III (light blue) of separase. 

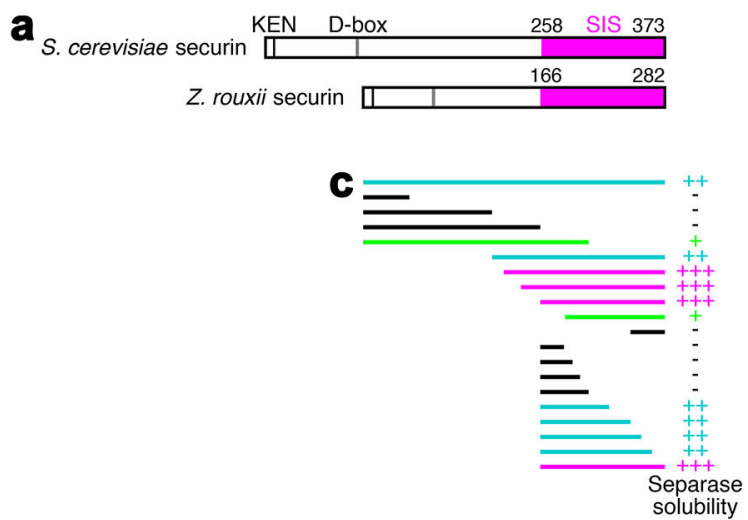

b

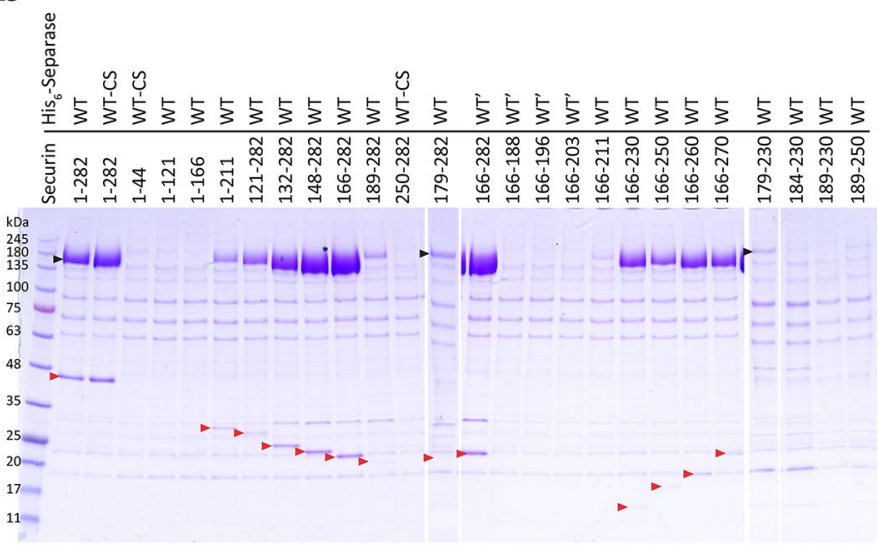

Figure 4.

Biochemical characterizations of the interactions between separase and securin. (a). Domain organizations of $S$. cerevisiae and $Z$. rouxii securin. The KEN motif and the destruction box (D-box) are also indicated. (b). Z. rouxii separase (with an N-terminal His tag) was coexpressed with various segments of $Z$. rouxii securin. The eluates from nickel columns were separated by SDS gel electrophoresis. The positions of separase and securin are indicated with the black and red arrowheads, respectively. WT: full-length $Z$. rouxii separase; WT-CS: full-length $Z$. rouxii separase $\mathrm{C} 1497 \mathrm{~S}$ mutant; $\mathrm{WT}^{\prime}: Z$. rouxii separase with an internal deletion of residues 952-1010, corresponding to a poorly conserved, disordered loop in domain IV of the $S$. cerevisiae separase structure. For gel source data, see Supplementary Figure 1. (c). Summary of the expression results in panel b. The solubility levels of separase for various securin segments are indicated. 